Experimental astronomy

DOI: $10.1007 / \mathrm{s} 10686-014-9382-5$

The final publication is available at http://link.springer.com

\title{
The effect of sideband ratio on line intensity for Herschel/HIFI
}

\author{
Ronan Higgins • David Teyssier • Colin \\ Borys • Jonathan Braine - Claudia \\ Comito - Bertrand Delforge • Frank \\ Helmich • Michael Olberg • Volker \\ Ossenkopf • John Pearson • Russell \\ Shipman
}

Received: date / Accepted: date

R. Higgins, C. Comito and V. Ossenkopf

I. Physikalisches Institut

Universität zu Köln

Zülpicher Strae 77

50937 Köln, Germany

Tel.: +49 2214701707 E-mail: higgins@ph1.uni-koeln.de

D. Teyssier

Herschel Science Centre

European Space Astronomy Centre (ESAC)

P.O. Box 78, 28691 Villanueva de la Cañada

Madrid, Spain

C. Borys

California Institute of Technology

1200 E California Blvd.

MS 100-22

Pasadena, CA, 91125, USA

J. Braine

Observatoire de Bordeaux, LAB

2 rue de l'Observatoire

BP 89, 33270 Floirac, France

B. Delforge, F. P. Helmich and R. Shipman

SRON Netherlands Institute for Space Research \& Kapteyn Astronomical Institute University of Groningen

PO-Box 800, 9700 AV Groningen

The Netherlands

M. Olberg

Onsala Space Observatory

Chalmers University of Technology

S-43992 Onsala, Sweden

J. C. Pearson

Mail Stop 301-429, Jet Propulsion Laboratory

4800 Oak Grove Drive

Pasadena, CA 91109, USA 
Abstract The Heterodyne Instrument for the Far Infrared (HIFI) on board the Herschel Space Observatory is composed of a set of fourteen double sideband mixers. We discuss the general problem of the sideband ratio (SBR) determination and the impact of an imbalanced sideband ratio on the line calibration in double sideband heterodyne receivers. The HIFI SBR is determined from a combination of data taken during pre-launch gas cell tests and in-flight. The results and some of the calibration artefacts discovered in the gas cell test data are presented here along with some examples of how these effects appear in science data taken in orbit.

Keywords HIFI · Herschel · Heterodyne - Calibration · Sideband ratio

\section{Introduction}

The Herschel Space Observatory ([21]) was launched on May 14th 2009 and successfully observed objects in the Sub-millimetre and Far Infrared (FIR) bands from the Solar System to the most distant reaches of the Universe. The mission ended on April $29^{\text {th }} 2013$ when the Helium coolant boiled off. Its spectral coverage was provided by three instruments, PACS, SPIRE and HIFI. PACS (Photodetecting Array Camera and Spectrometer) was an imaging camera and low-resolution integral field spectrometer covering wavelengths from 55 to $210 \mu \mathrm{m}$ 22]. SPIRE (Spectral and Photometric Imaging Receiver) was also a camera and a Fourier transform spectrometer (FTS) covering wavelengths from 194 to $671 \mu \mathrm{m}$ [8]. HIFI (Heterodyne Instrument for the Far-Infra-red) was a heterodyne detector and spectrometer providing high resolution spectroscopy capability over two continuous frequency ranges of $488-1272$ and $1430-1902 \mathrm{GHz}$ [3]. This work addresses the calibration of the HIFI instrument.

HIFI covers its spectral range using 14 heterodyne detectors, mixing down the FIR signal to radio frequencies. They are organised in 7 bands with 2 mixers each, sensitive to orthogonal polarizations. The mixers in each band are pumped by a pair of Local Oscillator (LO) chains, covering respectively the lower (LO chain $a$ ) and upper ( $\mathrm{LO}$ chain $b$ ) frequencies of a band tuning range. Table 1 provides an overview of the frequency coverage and mixer technologies used in HIFI.

Observing in the environment of space allows unobstructed by atmosphere coverage of the entire HIFI frequency range. It also removes the day night cycle constraints and allows for continuous visibility of a source over an observational day. From a calibration perspective, observing from space also gives a number of advantages over ground based telescopes. The absence of the atmosphere from the calibration equation removes one of the major sources of error in flux determination 9]. Owing to a careful thermal control of the detection chain, a high temperature stability is achieved, that reduces mixer gain variation, and returns better data quality with less baseline features such as standing waves and baseline distortions. However, at the shorter wavelengths of the Hot Electron Bolometer (HEB) mixers, data quality was at times poor due to system instability [11, 16].

In anticipation of this unique environment ambitious calibration accuracies were sought, with a baseline calibration uncertainty of $10 \%$ and a goal calibration uncertainty of $3 \%$ [23]. The main sources of calibration error inherent to a double sideband heterodyne system were determined to be the sideband ratio (hereafter 


\begin{tabular}{|l|l|l|l|l|l|}
\hline $\begin{array}{l}\text { Mixer } \\
\text { band }\end{array}$ & $\begin{array}{l}\text { LO Frequency } \\
\text { range (GHz) }\end{array}$ & $\begin{array}{l}\text { IF BW } \\
(\mathrm{GHz})\end{array}$ & $\begin{array}{l}\text { Detector } \\
\text { technology }\end{array}$ & $\begin{array}{l}\text { Beam } \\
\text { combiner }\end{array}$ & $\begin{array}{l}\text { Feed and coupling } \\
\text { structure }\end{array}$ \\
\hline $\mathbf{1}$ & $488-628$ & $4-8$ & SIS $^{a}[6]$ & $\begin{array}{l}\text { Beamsplitter } \\
\text { microstrip }\end{array}$ & $\begin{array}{l}\text { corrugated horn } \\
\text { and waveguide }\end{array}$ \\
\hline $\mathbf{2}$ & $634-794$ & $4-8$ & SIS $^{a}[25]$ & $\begin{array}{l}\text { Beamsplitter } \\
\text { microstrip }\end{array}$ & $\begin{array}{l}\text { corrugated horn } \\
\text { and waveguide }\end{array}$ \\
\hline $\mathbf{3}$ & $807-953$ & $4-8$ & SIS $^{a}[4]$ & $\begin{array}{l}\text { Diplexer } \\
\text { microstrip }\end{array}$ & $\begin{array}{l}\text { corrugated horn } \\
\text { and waveguide }\end{array}$ \\
\hline $\mathbf{4}$ & $957-1114$ & $4-8$ & SIS $^{a}[4]$ & $\begin{array}{l}\text { Diplexer } \\
\text { microstrip }\end{array}$ & $\begin{array}{l}\text { corrugated horn } \\
\text { and waveguide }\end{array}$ \\
\hline $\mathbf{5}$ & $1116-1272$ & $4-8$ & SIS $^{b}[15]$ & $\begin{array}{l}\text { Beamsplitter } \\
\text { microstrip }\end{array}$ & $\begin{array}{l}\text { lens and twin slot } \\
\text { planar antenna }\end{array}$ \\
\hline $\mathbf{6}$ & $1430-1698$ & $2.4-4.8$ & HEB $^{c}[2]$ & Diplexer & $\begin{array}{l}\text { lens and twin slot } \\
\text { planar antenna }\end{array}$ \\
\hline $\mathbf{7}$ & $1701-1902$ & $2.4-4.8$ & HEB $^{c}[2]$ & Diplexer & $\begin{array}{l}\text { lens and twin slot } \\
\text { planar antenna }\end{array}$ \\
\hline
\end{tabular}

Table 1 Overview of mixer technology, materials and implemented antenna technology [3]. ${ }^{a}$ $\mathrm{Nb}-\mathrm{Al}_{2} \mathrm{O}_{3}-\mathrm{Nb},{ }^{b} \mathrm{Nb}-\mathrm{AlN}-\mathrm{NbTiN},{ }^{c} \mathrm{NbN}$ Phonon cooled.

SBR), standing waves, and the calibration load coupling. Specific tests were implemented prior to launch to constrain these error sources. They are described in [27].

In this paper we discuss the sideband ratio derived from flight data and from the pre-launch test campaign conducted with a gas cell. Section 2 gives the background on the significance of the sideband ratio and Section 3 describes the various phenomena involved in defining its characteristics. Section 4 summarizes the results from the gas cell test campaign. Section 5 provides examples of how the SBR manifests in certain areas of the HIFI frequency range. A discussion section follows summarizing the lessons learned from this calibration effort.

\section{What is the sideband ratio and why is it important}

The HIFI mixers are double sideband (DSB) mixers which detect signals in an upper (USB) and lower (LSB) sideband simultaneously as the mixing process does not distinguish between positive and negative differences between sky frequency and LO frequency. The width of this sideband is the mixer IF (intermediate frequency) bandwidth (BW) which is dependent on the mixer design, see Table1 The detected signal is calibrated using the two-load calibration [20, 18]. This method requires the observation of a reference blank sky position, together with internal hot and cold sources. Since the temperature of the hot and cold loads and their coupling to the mixers are known, one can assign an intensity to the sky signal. Using this method one can determine the double sideband intensity of the detected signal. To accurately assign an intensity to a given spectral channel in a given sideband one must have an a priori knowledge of the fraction of the double sideband signal that belongs to the respective upper and lower sideband at a given LO frequency. This fraction is known as the sideband ratio.

The sideband ratio, $R$, is the ratio of one sideband gain over the other and defined as:

$$
R=\frac{\gamma_{u s b}}{\gamma_{l s b}}
$$




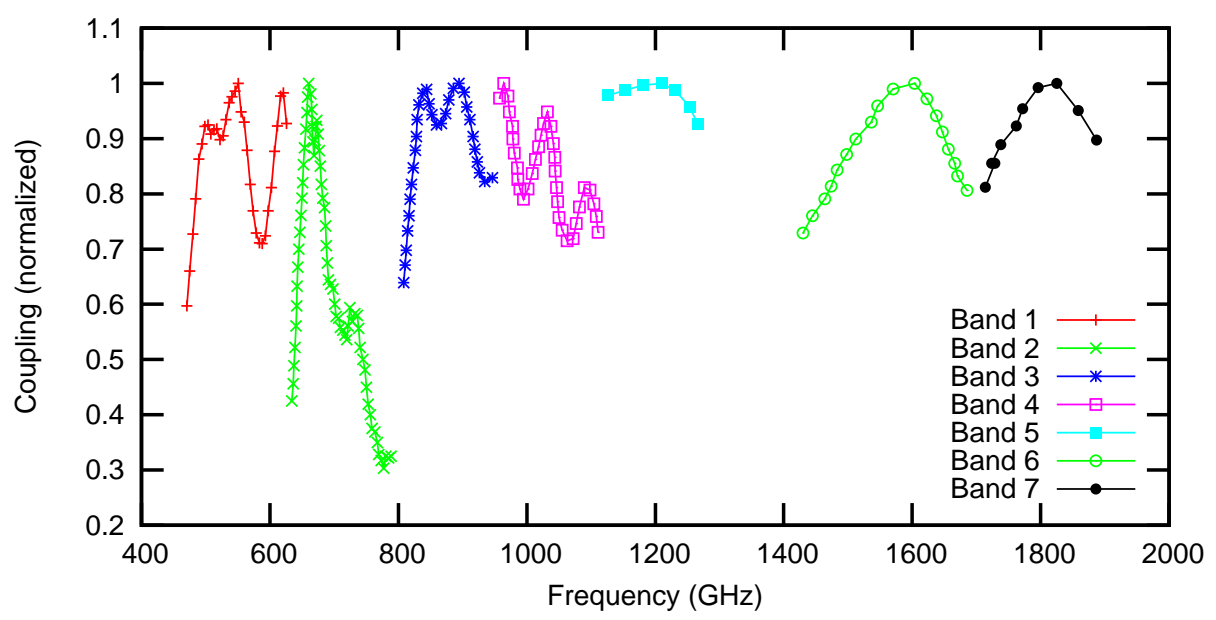

Fig. 1 Normalized mixer broadband coupling for bands 1-7 determined through FTS measurements with the mixer biased in direction detection mode. The data for band 1 are taken from [6], band 2 is from [25], bands $3 \& 4$ are from [4], band 5 is from [15] and the data for bands $6 \& 7$ are taken from [2].

where $\gamma_{u s b}$ is the response in the USB and $\gamma_{l s b}$ is the response in the LSB. The total instrument response, $\gamma_{d s b}$, is then:

$$
\gamma_{d s b}=\gamma_{u s b}+\gamma_{l s b}
$$

In the HIFI data processing pipeline a dedicated pipeline step doSidebandGain converts the double sideband intensity into separate upper and lower sideband intensities [13]. In this step the DSB spectra is divided by the normalized sideband ratio, $G_{u s b}$, to return the upper sideband intensity, and independently divided by the factor $1-G_{u s b}$ to return the lower sideband intensity. The normalized upper sideband gain is related to the sideband ratio, $R$, as follows:

$$
G_{u s b}=\frac{\gamma_{u s b}}{\gamma_{d s b}}=\frac{\gamma_{u s b}}{\gamma_{u s b}+\gamma_{l s b}}=\frac{R}{1+R}
$$

$G_{u s b}$ describes what fraction of the total double sideband response stems from the upper sideband. Then following from equation 2 $1-G_{u s b}$ is the fraction of that DSB response that comes from the lower sideband 1 .

In an ideal double sideband heterodyne receiver both sidebands have equal gain and hence $G_{u s b}$ is 0.5 . This is the base assumption for HIFI; however, as we will see in the following sections, the sideband ratio can vary considerably over a mixer band. 


\section{Reasons for sideband ratio imbalance}

\subsection{Mixer broadband coupling}

The degree of sideband imbalance is mainly driven by the variation of the mixer broadband coupling. This coupling was measured for all HIFI mixers prior to flight using an FTS. For this test, the FTS is coupled to the mixer and swept in order to extract the mixer output power at each frequency, and derive the mixer broadband coupling. It should be noted, however, that the FTS measurements are taken with the mixer biased to be sensitive to direct detection and not in heterodyne mode (i.e. the mixer is not pumped by an LO source). Fig. 1 provides an overview of the coupling measured for each HIFI mixer. It shows that mixers 1-4 have large variations in coupling compared to mixers $5-7$. The reasons for this vary. Mixers 3 and 4 were measured with the same experimental setup and were both affected by a non optimum cryostat window which introduced a large standing wave into the measured data. Bands 1 and 2 were taken under better conditions and provide a clearer picture of this coupling. Bands 5-7 show less frequency variation which is likely due to the coupling structure or mixer technology.

The FTS spectra shown in Fig. 1 can be converted into a sideband gain plot using equation 3 Fig. 2 shows an example of the variation in upper and lower sideband gain and the resulting sideband ratios in the HIFI band 2 for 3 different LO frequencies. The effect is most prominent in regions where there is a large slope in the broadband coupling. In these regions, such as the band edges, one sideband is dominant over the other. At the lower band edge e.g. the LSB coupling is less than the USB one and hence we have a normalized sideband ratio, $G_{u s b}$, higher than 0.5 . The opposite effect is seen at $750 \mathrm{GHz}$ where the USB coupling is weaker than that of the LSB and hence $G_{u s b}$ is less than 0.5. In regions, such as at 665 $\mathrm{GHz}$, where the coupling is equal in both sidebands we have a balanced sideband gain scenario, but still a small slope in the gain is seen over the IF bandwidth.

Returning to Fig. 1 one can expect that mixers 5-7 have less sideband ratio imbalance since the broadband coupling varies slowly with sky frequency compared with bands 1-4. Also from the Fig. 2 it should be noted that the sideband ratio is more extreme towards higher IF's since these regions are at the maximum frequency separation and so are more sensitive to slopes in broadband coupling. Since the HEB mixers have a lower IF than the SIS mixers, they are less sensitive to sideband ratio imbalances. This will be discussed further in section 5.5 .

\section{Impact on the science data}

Figure 3 provides an overview of the heterodyne detection process and the effect of an imbalanced sideband ratio on the spectral lines. Figure $3(\mathrm{a})$ shows an example of a simulated OCS spectrum between 712 and $730 \mathrm{GHz}$ with the LO tuned to $721.3 \mathrm{GHz}$. The two OCS lines seen in the figure are at 716.546 and $728.654 \mathrm{GHz}$ which results in the down converted lines appearing at 4.75 and $7.354 \mathrm{GHz}$ respectively in the IF band. The resulting IF spectra are shown in Fig. 3(b) for the

\footnotetext{
1 In case of LO impurities, providing coupling to multiple frequencies, the heterodyne receiver can also be considered as a multi-sideband receiver where the normalized gains of all sidebands add up to unity
} 


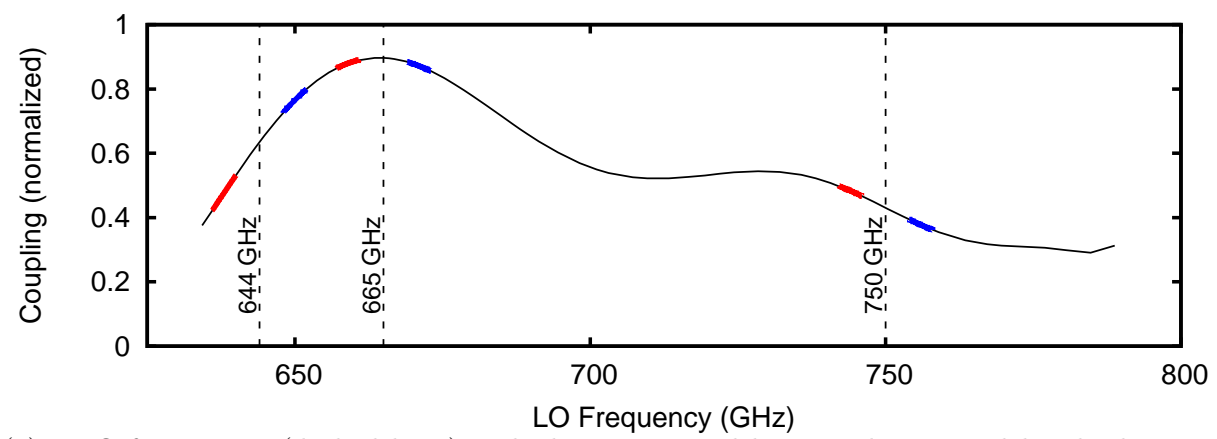

(a) 3 LO frequencies (dashed lines) with their associated lower and upper sidebands shown in red and blue respectively on top of the band 2 mixer broadband coupling measurement.
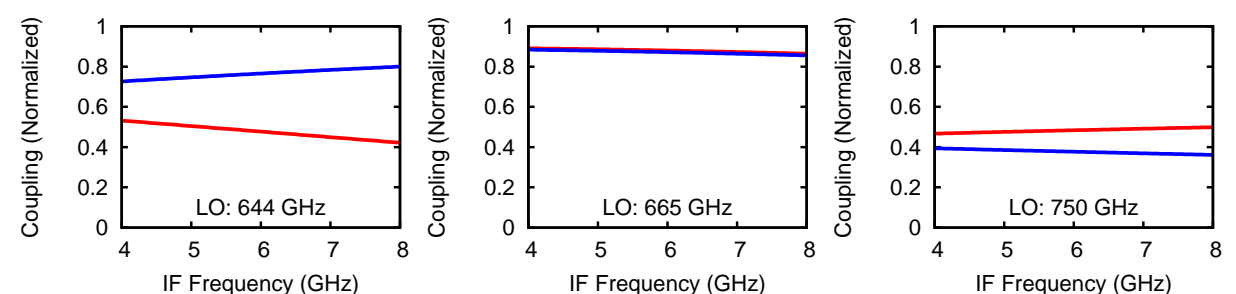

(b) Upper (blue) and lower (red) sideband mixer response at different LO frequencies plotted against IF.
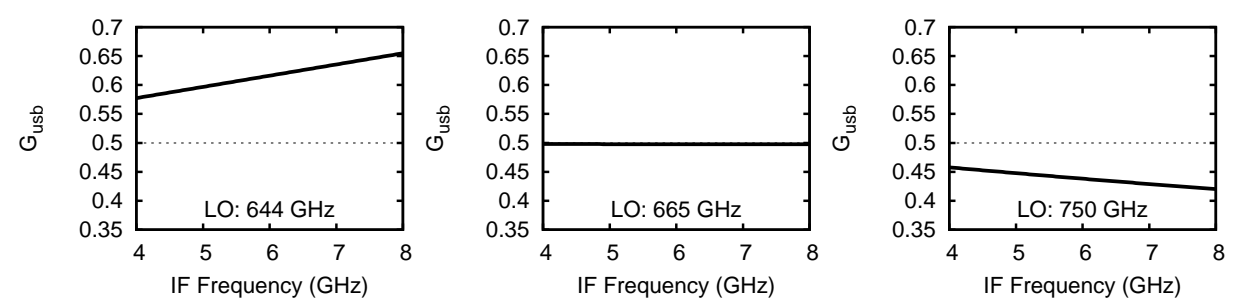

(c) Resulting sideband ratio, $G_{u s b}$.

Fig. 2 Sideband ratio, $G_{u s b}$, calculated from FTS broadband coupling measurements in band 2 .

USB-dominated and balanced sideband ratio scenarios illustrated in Fig. 3(c) The intensity in the down converted data are approximately half the original intensity, this is known as the double sideband intensity. This is similar to the level 1 data from the HIFI data processing pipeline 13].

This difference in double sideband and single sideband intensity is a result of the calibration process. Since the data are intensity calibrated by observing a hot and cold load the final spectrum is a fraction of the difference of their signals. Since the internal loads provide broadband signals and contribute to both sidebands a nearly equal continuum emission, a spectral line signal observed in only one sideband is calibrated as approximatively half the intensity it has in reality.

Figure $3(\mathrm{a})$ shows equal intensities for the simulated OCS lines. In the down converted DSB spectrum shown in Fig. 3(b), the USB-dominated sideband ratio 


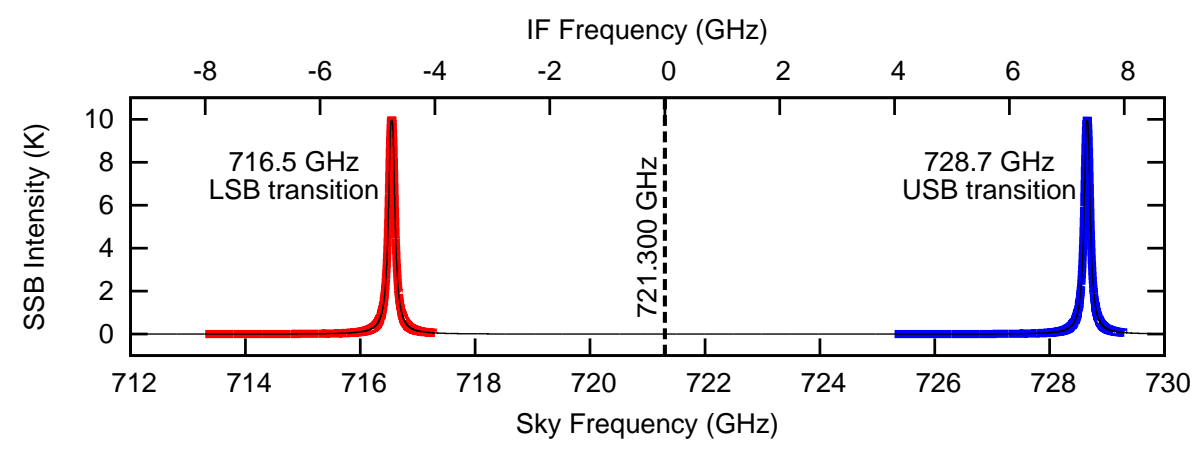

(a) Simulated OCS spectrum shown between 712 and $730 \mathrm{GHz}$. The resulting upper and lower sidebands components are highlighted in blue and red respectively when observing with an LO frequency of $721.3 \mathrm{GHz}$ and an IF bandwidth from 4 to $8 \mathrm{GHz}$.

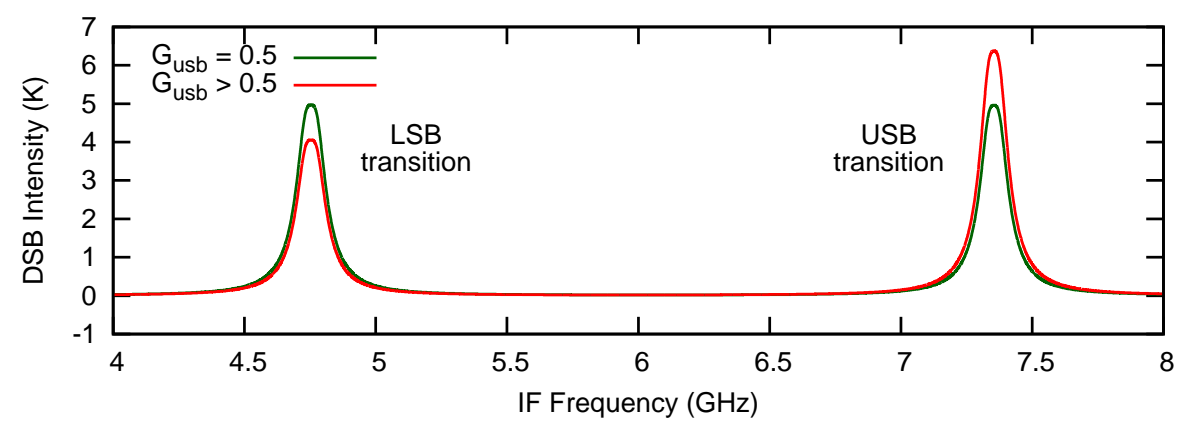

(b) Simulated HIFI double sideband spectrum applying the USB-dominated sideband ratio at $644 \mathrm{GHz}$ from Fig. 3(c) (red) and a balanced sideband ratio scenario (green).

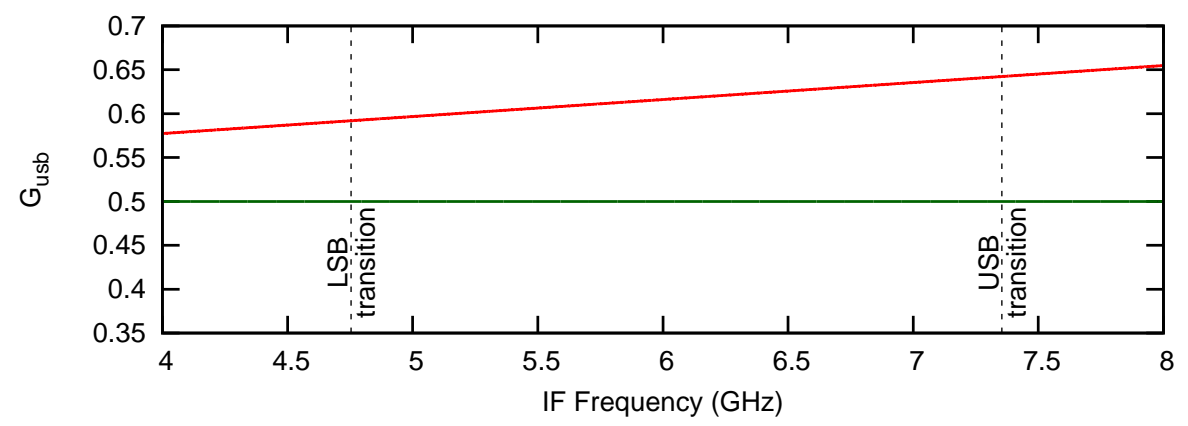

(c) Sideband ratio versus IF at $644 \mathrm{GHz}$ from Fig. 2(c) (red) compared with a sideband ratio balanced scenario where $G_{u s b}$ is 0.5 across the IF band (green).
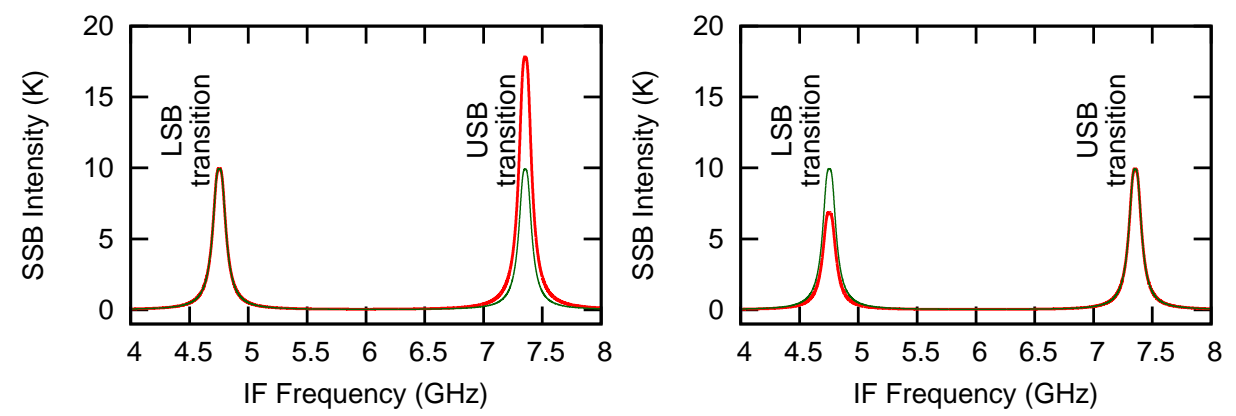

(d) Left: Lower sideband intensities generated by scaling double sideband data in Fig. 3(b) with $G_{l s b}=\left(1-G_{u s b}\right)$ values shown in Fig. 3(c) Right: Upper sideband intensities generated by scaling with $G_{u s b}$. Note that the left panel shows the correct amplitude only for the LSB transition and the right panel only for the USB transition.

Fig. 3 The effect of a sideband imbalance on the double sideband line intensity for the molecule OCS observed at an LO frequency of $721.3 \mathrm{GHz}$. The figure highlights the need to apply the correct sideband scaling factor to the appropriate spectral line. 
example $\left(G_{u s b}>0.5\right)$ shows a noticeable difference in line intensity between the upper and lower sideband spectral lines. This is the effect of the sideband ratio imbalance. Where the sideband ratio is 0.5 the lines have equal intensities and are half the original SSB intensities. This example also emphasises how the SBR can noticeably vary over the IF bandwidth when a large imbalance exists in the mixer response on short frequency scales.

Figure $3(\mathrm{~d})$ shows an example of simulated level 2 data from the HIFI pipeline with the sideband gain ratio applied. This example highlights the importance of applying the LSB or USB sideband ratio to the respective LSB or USB line(s). When the appropriate sideband ratio is applied to the appropriate line, the single sideband line intensity can be fully recovered. Without this a priori sideband ratio knowledge assigning the correct line intensity would not be possible.

\subsection{Diplexer effect on the sideband ratio}

The LO and sky beam coupling for bands 3, 4, 6 and 7 was achieved through a Martin-Puplett diplexer (bands 1, 2 and 5 used a beamsplitter approach). The advantage of the Martin-Puplett diplexer is that the amount of LO power reaching the mixer at the correct polarization angle can be maximised. Each LO frequency has a unique diplexer position that has the maximum LO path transmission at the LO frequency and the maximum sky path transmission peaking at the IF bandwidth centre, see Fig. 4(a) The process of setting the diplexer position is described in more detail in $[\overline{19}]$.

The diplexer can have an influence on the sideband ratio if the optimum position is not used for a given LO frequency. Ideally the respective sideband coupling through the diplexer should be symmetric, see Fig. 4(a) however, a slight imbalance can lead to a sideband ratio slope occurring across the IF bandwidth, most prominently towards band edges, see Fig. 4(b) Fig. 4(c) shows an example of the ${ }^{12} \mathrm{CO}(8-7)$ transition measured during gas cell tests at different LO frequencies in the lower sideband. The slope in line intensity induced by a diplexer mistune is noticeably across the IF band.

These effects were seen in the band 3 and 4 gas cell test data, and are particularly noticeable in saturated $\mathrm{H}_{2} \mathrm{O}$ lines where the broad and flat saturated line plateau had a significant slope indicating a diplexer mistune 10]. This diplexer mistune introduced an additional uncertainty into the sideband ratio derived from the gas cell tests. Fortunately these diplexer tuning effects have not been observed in flight, as a new and improved diplexer tuning model was generated based on the ground testing experience. Investigation of ${ }^{12} \mathrm{CO}(8-7)$ transition in orbit shows no evidence of the variation shown in Fig. 4(c). However, further data mining is required for a firm confirmation that we have no diplexer induced sideband ratio effect due to mistuning.

In addition to the diplexer mistuning possibilities, the diplexer can also introduce complicated standing wave patterns. This was investigated by Siebertz et al. 24] using a band 2 prototype mixer. They showed that there can be significant standing waves issues at the IF band edges, which modulate the line intensity. Delforge et al. [5] have underpinned the experimental work with a theoretical frame work. Due to the coarse frequency sampling of the standard spectral scan 

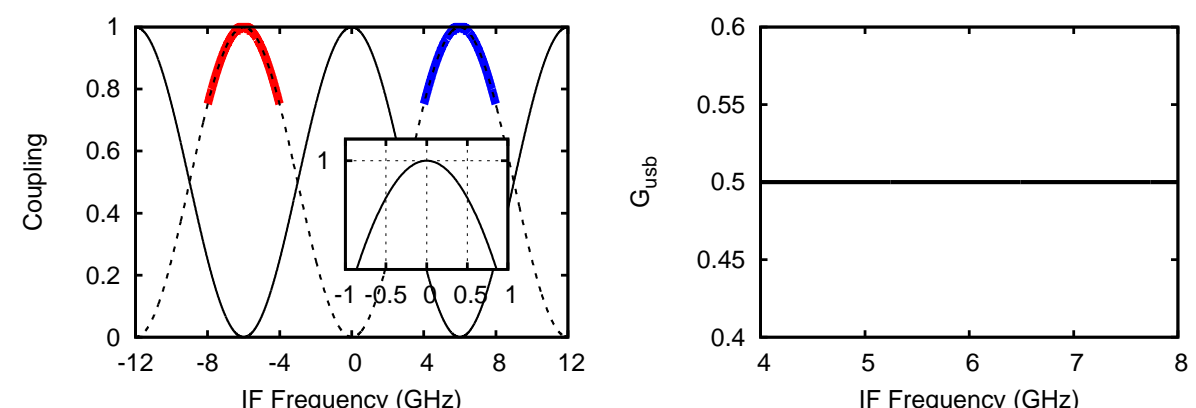

(a) Left: Correctly tuned diplexer coupling. The solid line shows the LO signal coupling, the dashed line shows the sky signal coupling. The red and blue thick lines indicate the IF bandwidth of the respective LSB and USB. Right: Sideband ratio for a correctly tuned diplexer.
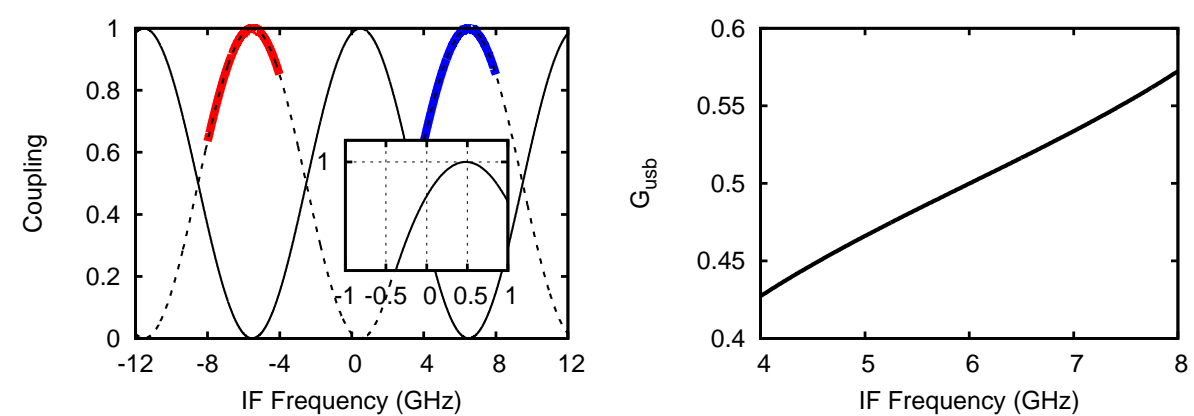

(b) Left: Diplexer coupling for a $-10 \mu \mathrm{m}$ mistune. Right: Sideband ratio for a $-10 \mu \mathrm{m}$ mistuned diplexer showing the IF-dependent slope.

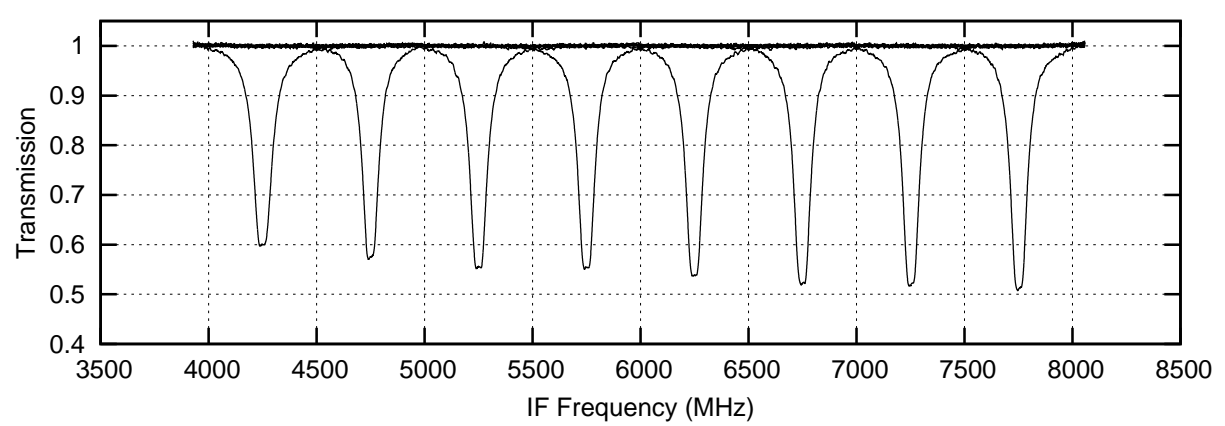

(c) ${ }^{12} \mathrm{CO}$ (8-7) gas cell test data measured in the lower sideband at multiple LO frequencies showing evidence of a $-10 \mu \mathrm{m}$ mistuned diplexer. Since the line is measured in the lower sideband the applicable sideband ratio is one minus that shown in Fig. 4(b)

Fig. 4 The effect of diplexer mistune on the sideband ratio.

used during the HIFI observations, it is difficult to measure the influence of standing waves. However, a number of dedicated tests were undertaken towards the end of the mission in order to measure the influence of this effect. These tests involved placing a spectral line towards the IF band edges and observing the line at different $\mathrm{LO}$ frequencies separated by tens of $\mathrm{MHz}$ and different diplexer mistunings. 
These tests will help established the degree of scatter due to standing waves and diplexer mistuning.

\subsection{LO spectral purity}

It has been demonstrated that some frequency areas over the HIFI tuning range are affected by spectral impurity. What this means is that the DSB spectrum obtained from the mixing process may hold signal arising from frequency ranges not belonging to the intended ones. In this situation, the detector gain cannot be described with only 2 components (LSB and USB) but has to take into account an additional component (the Outer Sideband contribution, OSB). In practice this implies that the total system gain is altered by that of the unwanted regions and the line calibration becomes erroneous as it assumes a sideband correction relying on only two sidebands.

One can extend the formalism introduced in equations 2 and 3 as follows. Assuming that the OSB contributes to the total gain as $\gamma_{o s b}$, the new sideband gain ratio correction to apply to lines from the USB writes:

$$
G_{u s b}^{\text {impure }}=\frac{\gamma_{u s b}}{\gamma_{u s b}+\gamma_{l s b}+\gamma_{o s b}}
$$

Since we calibrate against continuum sources (internal loads) this will manifest in a decrease of the sideband gain ratio by a factor $F_{\text {corr }}(<1)$ such that $G_{\text {usb }}^{\text {impure }}=$ $G_{u s b} \times F_{\text {corr }}$. Consequently, one can see that $\gamma_{u s b}+\gamma_{l s b}+\gamma_{o s b}=\left(\gamma_{u s b}+\gamma_{l s b}\right) / F_{\text {corr }}$, so that the new sideband ration correction for lines in the LSB will now write:

$$
\frac{\gamma_{l s b}}{\gamma_{u s b}+\gamma_{l s b}+\gamma_{o s b}}=\left(1-G_{u s b}\right) \times F_{c o r r}
$$

i.e. the correction factor applies similarly to lines in either sideband.

The most severe impure region was identified in band 5b, which was in fact only released to the observer community after most of its purity issues got resolved. Evidence of the sideband ratio imbalance in this band can be seen in the gas cell tests shown in Fig. 5. In this example one can see that depending on which sideband the line is observed in, the intensity can be greatly over- or underestimated. Some other spectral ranges were purified during the mission, in particular in band $5 \mathrm{a}$ and in band $3 \mathrm{~b}(951-953 \mathrm{GHz})$.

\section{The gas cell test campaign}

Dedicated gas cell measurements were performed prior to launch to measure the HIFI sideband ratio. The test apparatus is discussed in detail in 26], while the data acquisition and process of extracting the sideband ratio from the gas cell test data is detailed in [12, 10]. The HIFI gas cell was designed to present a saturated column of ${ }^{12} \mathrm{CO},{ }^{13} \mathrm{CO}$, OCS and $\mathrm{H}_{2} \mathrm{O}$ gas to the instrument detectors. By measuring these gases at various LO frequencies a sparsely sampled picture of the sideband ratio was generated. Fig. 5 gives an overview of the sideband ratios derived from the gas cell tests.

It is interesting to compare the FTS measurements shown in Fig. 11 with the sideband ratio measured during the gas cell tests. As shown in Fig. 2 it is possible 

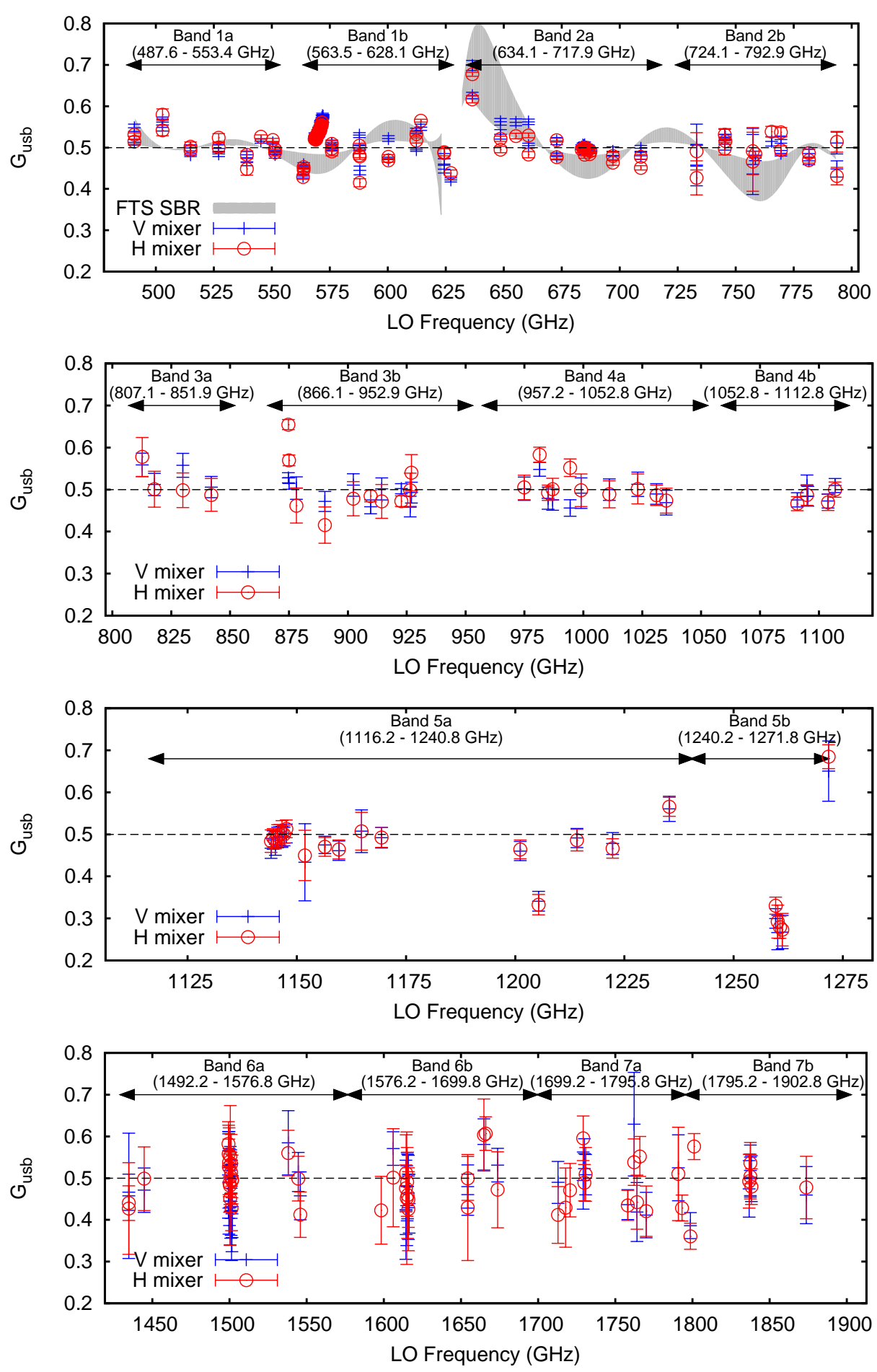

Fig. 5 Overview of sideband ratio for all $\mathrm{H}$ and $\mathrm{V}$ mixers measured using the gas cell test setup [10]. A derived sideband ratio extracted from FTS measurement in Fig. 1 is plotted in gray for bands 1 and 2 . The gray area shows the maximum and minimum normalized sideband ratio calculated. 
to generate a sideband ratio from the FTS test data. There are, however, several limitations to this exercise: while the band 3 and 4 FTS measurements were affected by the FTS setup problems mentioned in Section 3.1 the gas cell test data shown for band 5 was affected by an impure LO signal (discussed in Section 3.3). Furthermore, the large scatter in the gas cell test data seen in bands 6 and 7 is due to poor data quality during the ground test campaign, see Section [5.5 and 11] for further details. This leaves bands 1 and 2 with the best combination of good FTS and gas cell test data.

The calculated sideband ratio extracted from the FTS measurements for bands 1 and 2 is plotted in gray behind the gas cell test results in Fig. 5 The gray region maps out the maximum and minimum normalized sideband ratio across the IF bandwidth for each LO tuning. The plots shown in Fig. 2(c) would correspond to a vertical slice of the gray FTS measurement region. From the plots there is some agreement between the FTS measurement and the sideband ratio inferred from the gas cell tests, in particular in the lower end of band $2 \mathrm{a}$ and upper end of band $1 \mathrm{~b}$, i.e. those areas mapping the expected drop in the mixer response towards the band edge. However, the regions associated with both ${ }^{12} \mathrm{CO}$ transitions at 576 and $691 \mathrm{GHz}$ cannot be reconciled in the two datasets.

In the following sections we show that several of the regions of sideband ratio imbalance predicted by the gas cell tests are also seen in the flight data, which leads to the conclusion that the gas cell measurements should be more representative of the actual sideband ratio affecting the science data. We recall that the FTS measurements are performed with an un-pumped mixer, so there could be additional mechanisms other than what the FTS broadband coupling measurement can reveal, such as an LO frequency dependent heterodyne IF conversion efficiency. Some regions of sideband ratio indeed show extreme effects that contradict the assumptions made so far in this paper. The ${ }^{12} \mathrm{CO}(5-4)$ region in band 1 for example (see Section 5.1) shows a sideband ratio that increases towards lower IF's in both sidebands, which is opposite to the behaviour shown in the leftmost example of Fig. 2(c)

\section{Sideband ratios measured in gas cell and science data}

5.1 Band 1: ${ }^{12} \mathrm{CO}(5-4)$

Figure 6(a) shows the sideband ratio extracted from gas cell test data between LO frequencies of 550 and $585 \mathrm{GHz}$. Noteworthy here is the similarity in sideband ratio between the $\mathrm{H}$ and $\mathrm{V}$ mixer. In this plot we see the sideband ratio extracted using three different molecules, $\mathrm{H}_{2} \mathrm{O},{ }^{12} \mathrm{CO}$ and OCS. From the figures one can see that depending on which LO frequency the ${ }^{12} \mathrm{CO}$ line is measured at there is a noticeable change in sideband ratio and therefore line intensity. Unfortunately the ${ }^{12} \mathrm{CO}$ (5-4) transition was only measured in the upper sideband in the gas cell tests. We can, however, use flight data from the upper and lower sideband and extrapolate the sideband ratio measured during the gas cell tests from the upper sideband into the lower sideband.

Figure 6(b) shows an example of the ${ }^{12} \mathrm{CO}$ line from OMC-2 FIR 4 taken as part of the CHESS key program[14]. The figure shows multiple observations of the ${ }^{12} \mathrm{CO}$ line at different LO frequencies and reveals a $\sim 10 \%$ scatter in line intensity. 


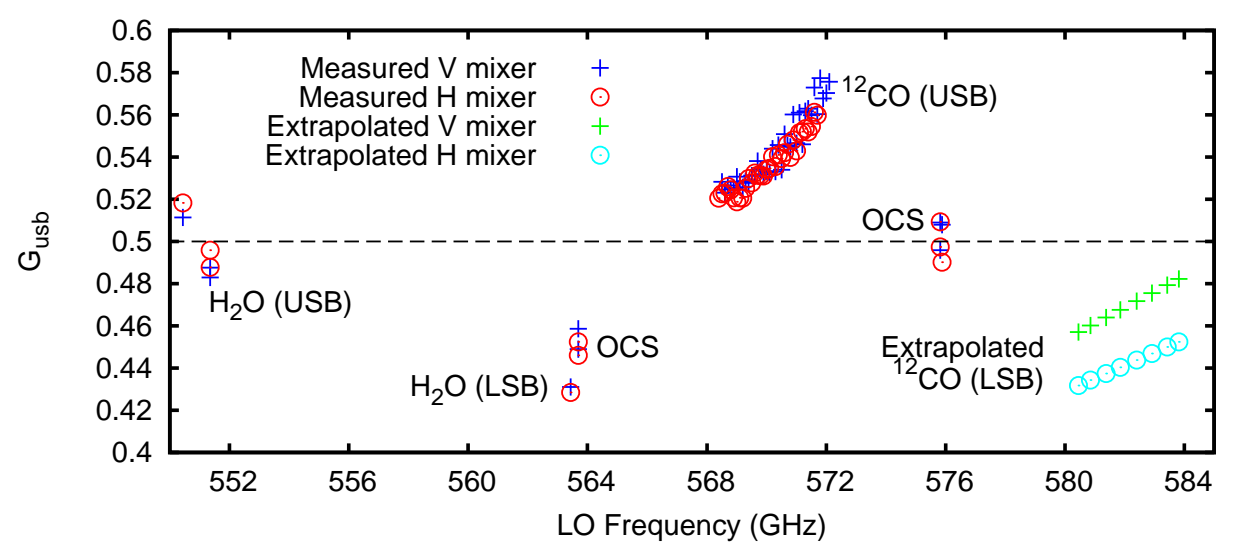

(a) Sideband ratio for the $\mathrm{H}$ and $\mathrm{V}$ mixers in the frequency range of the ${ }^{12} \mathrm{CO}(5-4)$ and $\mathrm{H}_{2} \mathrm{O}$ $557 \mathrm{GHz}$ transitions. The $\mathrm{LO}$ frequency range from $580-584 \mathrm{GHz}$ covers the ${ }^{12} \mathrm{CO}$ line as seen from the lower sideband, but it was not measured during the gas cell tests. The data shown are extrapolated from the upper sideband line using flight data to determine the relative trend.
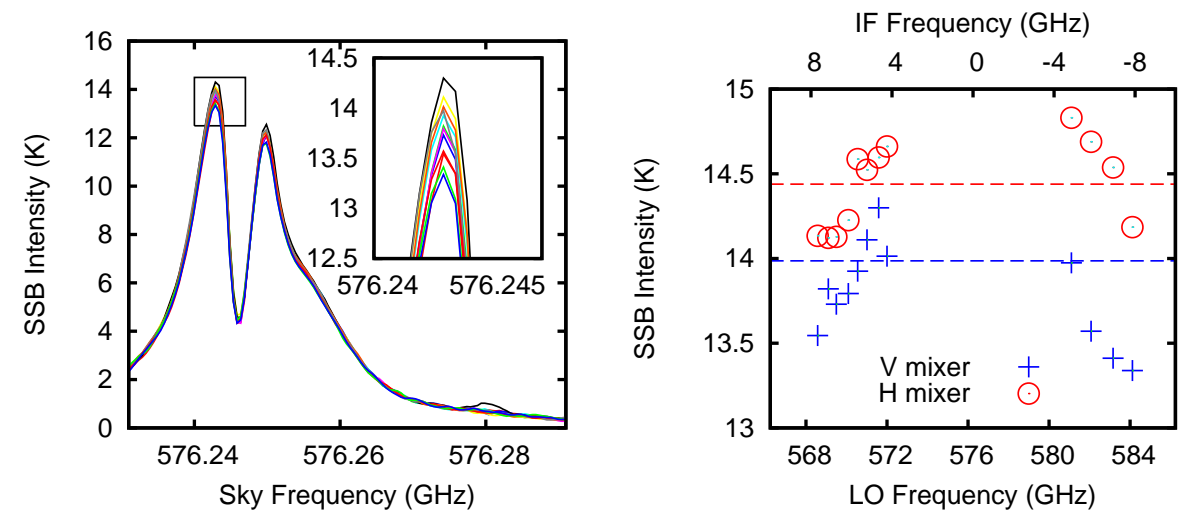

(b) Left: ${ }^{12} \mathrm{CO}$ (5-4) line measured with the V mixer on OMC-2 FIR 4 at different LO frequencies covering the LSB and USB showing a $\sim 10 \%$ peak intensity scatter. Right: $\mathrm{H}$ and V line peak intensity versus IF for lines shown in the left panel. The dashed lines show the peak intensity of the $\mathrm{H}$ and $\mathrm{V}$ deconvolved spectral lines. The DSB line intensity was converted to SSB assuming $G_{u s b}=G_{l s b}=0.5$.

Fig. 6 A comparison between the sideband ratio measured from gas cell tests and line intensity trends with LO frequency extracted from in flight spectral scans for the ${ }^{12} \mathrm{CO}(5-4)$ region. Similar trends are seen in both datasets.

When these intensities are plotted as a function of LO frequency the variation in the upper sideband trend is consistent with the trend measured in the gas cell test data. Using the spectral survey data from the upper sideband the intensity variation into the lower sideband can be examined. The data from Fig. 6(b) indeed shows that there is a similar IF-dependent trend in the lower sideband as the line intensity is seen to increase towards lower IF (points at negative IF). Using the gas cell measurements of the upper sideband behaviour of the ${ }^{12} \mathrm{CO}$ line in combination with the spectral scan data it is possible to extrapolate the sideband ratio into the region where the ${ }^{12} \mathrm{CO}$ transition is in the lower sideband ( $\mathrm{LO}$ frequencies 
between $580-584 \mathrm{GHz}$ ). The extrapolation is undertaken by correcting the upper sideband line with the correct sideband ratio. In the example shown the sideband corrected peak line intensity is estimated to be $13.5 \mathrm{~K}$ for the $\mathrm{H}$ mixer and $13 \mathrm{~K}$ for the $\mathrm{V}$ mixer. Using this intensity as a reference, the lower sideband ratio in the $580-584 \mathrm{GHz}$ can be derived. The extrapolated sideband ratios are shown in Fig. 6(a) From the plot it is apparent that an abrupt turnover in sideband ratio is required in order to match the upper sideband intensity to the lower sideband one. This turnover is supported by the OCS observation at an LO frequency of $\sim 576$ $\mathrm{GHz}$ which suggests a nearly balanced sideband ratio of 0.5 at an LO frequency between the two sidebands where the ${ }^{12} \mathrm{CO}$ transition is measured.

The ${ }^{12} \mathrm{CO}$ region we have discussed here serves to illustrate the complexity of the sideband ratio problem. Fig. 6(a) evidences that there is a certain level of structure that is not accurately probed between the spot frequencies collected in the gas cell test campaign. The first approach to the gas cell test results was to simply fit a polynomial through the measured sideband ratio and then interpolate between the measured points. The example shown here illustrates the futility of such an approach. It was initially assumed, based on the FTS measurements, that the sideband ratio would be a smooth function across the band; however, this is not the case everywhere. Further investigation is needed to explain the sharp features that are seen in the data. The next section examines another region of band 1 which is of particular importance to the HIFI science goals and shows a similar sharp sideband ratio feature.

\subsection{Band 1: $\mathrm{H}_{2} \mathrm{O}$ at $557 \mathrm{GHz}$}

The observation of water was one of the main science goals of HIFI. The ground state water lines occur around $557 \mathrm{GHz}$ (ortho- $\mathrm{H}_{2} \mathrm{O}$, band 1) and $1.11 \mathrm{THz}$ (para$\mathrm{H}_{2} \mathrm{O}$, band 4). The $557 \mathrm{GHz}$ water line covers the boundary between the upper and lower LO bands of the band 1 mixer. The lower region is known as LO band 1a and observes the line in the USB, while the upper region is the LO band $1 \mathrm{~b}$ and observes the line in the LSB. From the observation of a single water line alone various properties can be extracted; however, interestingly from the ratio of the ortho-to-para line intensity the physical conditions when the gas was formed can be determined [17]. In this section we will discuss the ortho- $\mathrm{H}_{2} \mathrm{O}$ line in band 1 .

Figure 7 shows an example of the deconvolved $557 \mathrm{GHz}$ line observed in both upper and lower sidebands using the $\mathrm{H}$ mixer as part of the CHESS key program. The line observed in the USB is $10 \%$ stronger than that observed in the LSB. This is consistent with the sideband ratio measured during the gas cell tests shown in Fig. 6(a) When the sideband ratio derived from the gas cell tests is applied to the respective USB and LSB measured lines, the discrepancy between the USB and LSB line intensity is greatly reduced. Much like the ${ }^{12} \mathrm{CO}$ region discussed in the previous section this extreme change in sideband ratio is not predicted by the FTS measurements and requires a rather extreme variation in broadband coupling to reproduce such an effect.

What is interesting in this example is that the line intensity is overestimated both when it is the upper and lower sideband. When the appropriate sideband ratio is applied to the respective sideband measurements, the line intensity is reduced for both upper and lower sideband lines (Fig. 7). Without knowledge of 


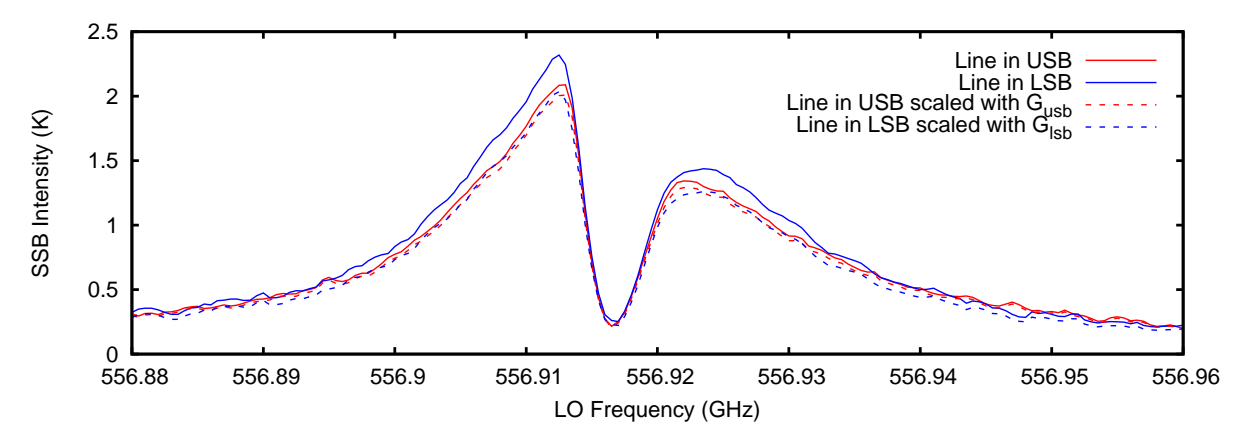

Fig. 7 Sideband ratio imbalance seen in $557 \mathrm{GHz}$ o- $\mathrm{H}_{2} \mathrm{O}$ line from OMC-2 FIR 4 [14]. The deconvolved line profile from band $1 \mathrm{a}$ (red) and $1 \mathrm{~b}$ (blue) is shown here. The line before sideband ratio correction is shown with the solid line, the dashed line shows the corrected line profile.

the sideband ratio, a standard approach here would be to average the two line profiles together which would result in an overestimated line intensity.

For further information on the application of sideband ratios after the pipeline processing see reference [28]. This technical note contains a table of sideband ratios at spot frequencies and describes how to apply the sideband ratio to level 2.

\subsection{Band 2 : lower band edge}

One of the most noticeable sideband ratio variations can be observed at the lower end of band $2 \mathrm{a}$. In this range, the mixer response is dropping steeply downwards of $650 \mathrm{GHz}$ and the imbalance between the two sideband gains increases accordingly, leading to an upper sideband being more efficient than the lower sideband. This mixer response drop is well measured by the gas cell test data obtained in this frequency range, as can be seen in Fig. 5(a). The grey area in this plot also shows that the trend is consistent with the FTS broadband coupling measurement 25. This strong imbalance is illustrated in Fig. 8] showing a gas cell measurement of two OCS subsequent transitions present in the respective sidebands at an LO frequency of $636.4 \mathrm{GHz}$. In a sideband-balanced scenario the OCS transitions should have similar line intensities, however the line from the LSB exhibits a much weaker absorption than its USB counterpart (note that both lines are saturated). This measurement also demonstrates that, in regions of steep mixer gain, the sideband ratio also has a noticeable IF-dependency, scaling typically with the ratio applying at the centre of the IF (see also Appendix A in [23]). The spectrum measured with the gas cell provide a real world example of the simulation described in Fig. 3 ,

\subsection{Band 5}

In the mixers used for the HIFI band 5, the coupling between the antenna and the SIS devices is effectively a resistor (normal metal) where there is a slow but increasing loss with frequency. This response characteristic is reflected in the system noise temperature of the mixer, whereby a constant slope is observed over 


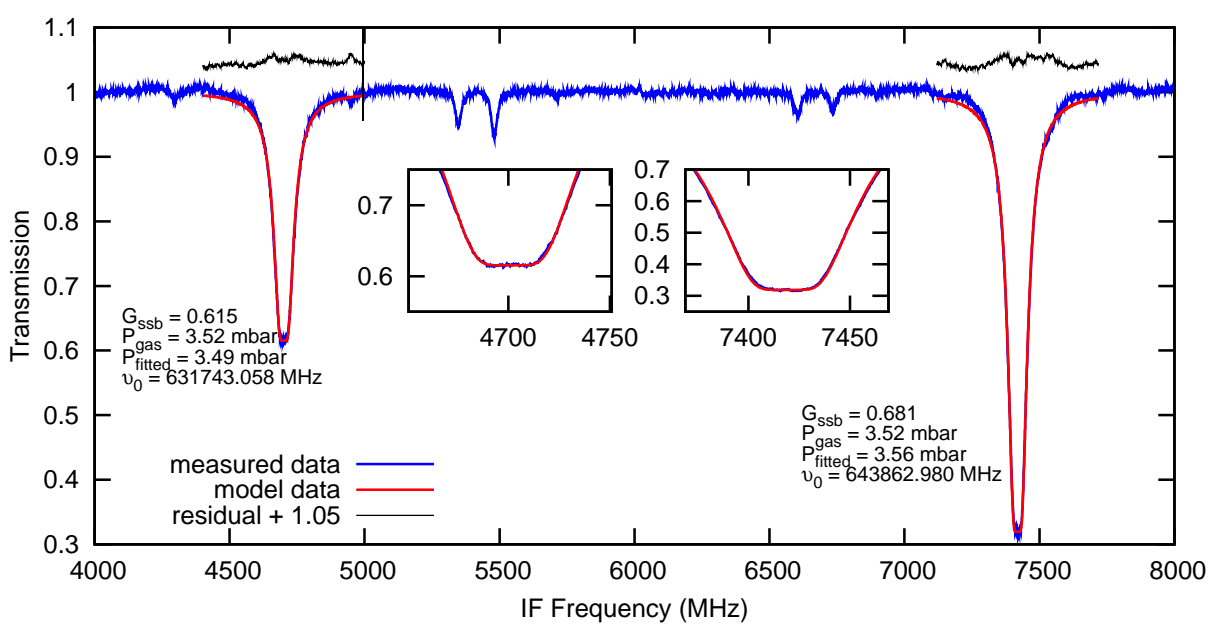

Fig. 8 Gas cell measurement of the $J=52-51$ and $J=53-52$ transitions of OCS in the HIFI Wide-Band-Spectrometer $\mathrm{H}$ at $\mathrm{LO}=636.444 \mathrm{GHz}$. The red profiles correspond to the best LTE (Local Thermal Equilibrium) fits - note that both lines are predicted to be saturated. The SBR at the IF centre is expected to be $G_{u s b}=0.652$; however, the values derived from the respective transitions at the two IF borders differ from this due to the additional IF-dependent slope.

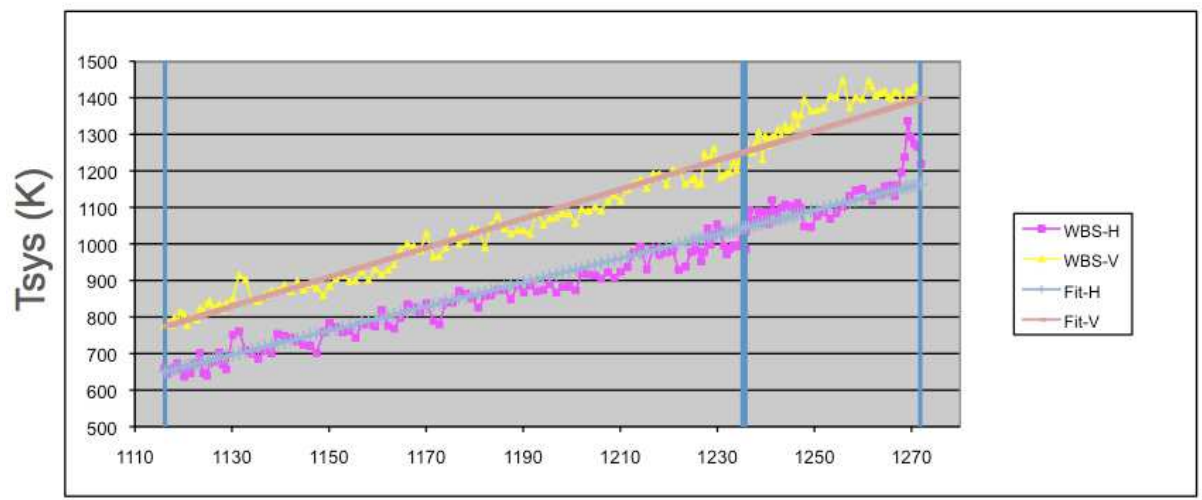

$\mathrm{LO}(\mathrm{GHz})$

Fig. 9 System noise temperature (DSB) for the $\mathrm{H}$ and $\mathrm{V}$ polarisations of the band 5 mixers. The vertical bars indicate the ranges for the LO band $5 \mathrm{a}$ and $5 \mathrm{~b}$ respectively. The straight lines correspond to a constant slope of $6 \%$.

the whole operational range, see Fig. 9] We have interpreted this behaviour as a systematic loss in the RF (Radio Frequency) input of the USB relative to that of the LSB. With an estimated slope around $6 \%$, the corresponding normalised sideband ratio for a line in the USB is $G_{u s b}=0.485$.

While the limited number of points from the gas cell test data in band 5 suggests a fairly constant sideband ratio, potentially below 0.5 within the error, checks on selected strong lines observed in orbit in both sidebands confirm this trend overall. 


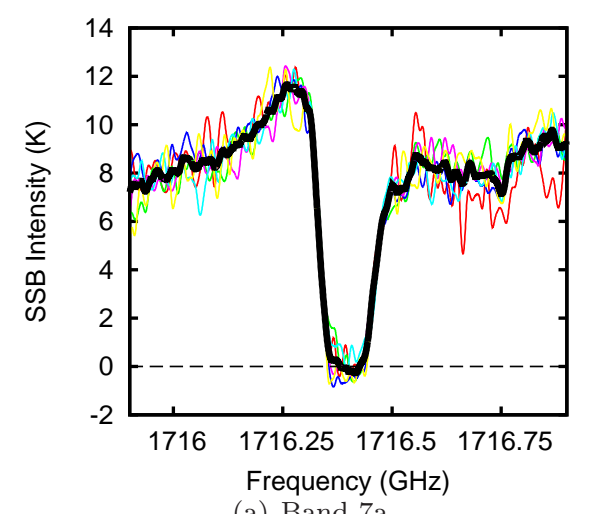

(a) Band 7a

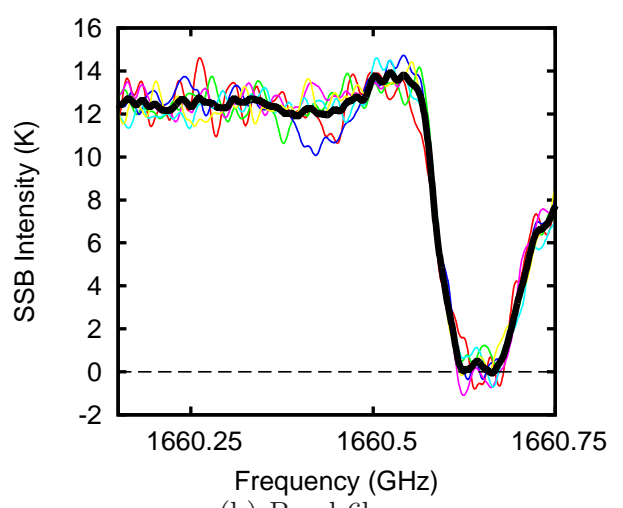

(b) Band $6 \mathrm{~b}$

Fig. 10 Saturated absorption lines seen in the $H$ polarization towards SgrB2(M) in bands 7a and $6 \mathrm{~b}$. The dark line is the average of more than 8 line profiles measured at different IF's in both the upper and lower sidebands.

\subsection{Bands $6 \& 7$}

As summarized in Table 1, the HEB mixers in bands 6 and 7 have a smaller bandwidth of $2.4 \mathrm{GHz}$, centered at $3.6 \mathrm{GHz}$, compared with the SIS mixer of bands 1-5. From Fig. 2] it is apparent that lower IF's are less sensitive to sideband ratio imbalances than higher ones. Furthermore the HEB mixer unit has an inherently flatter response due to the mixer and double slot antenna technology used (similar antennae are used in band 5). This is also noticeable from the FTS measurements in Fig. 1 which shows a slowly varying broadband coupling across the mixer range.

Nevertheless, the sideband ratio measured in the HEB bands during the gas cell tests, and summarized in Fig. 5 show a large degree of scatter. In the original HIFI calibration paper 23], the sideband ratio error was taken as the standard deviation of the sideband ratios across a given band, which has a large impact on the overall calibration accuracy of the HEB bands. However, when comparing flight data to gas cell test data, we see none of this scatter.

Figure 10 shows an example of two saturated absorption lines seen towards SgrB2(M), near the Galactic Centre. In this example a foreground cool gas cloud absorbs all the continuum background radiation at the observed molecular transition frequency. The spectra are plotted on a single sideband intensity scale and hence, for a fully saturated line the minimum is seen at $0 \mathrm{~K}$, indivative of a balanced sideband ratio scenario. The data shown here is taken from a spectral scan observation, so that the line is observed at different LO frequencies and hence different IF's. Spectra from both the upper and lower sidebands are overplotted and there is no discernible difference in line intensity, providing further evidence of a flat mixer gain at the scale of the IF bandwidth.

Returning to the gas cell test data, it was clear that the experimental set up used to measure the sideband ratio in the HEB bands was not representative of the way the data were going to be taken in flight [7]. The LO unit was mounted in a separate cryostat to the main HIFI unit, resulting in a degraded system stability due to path length modulation between the two cryostats. With the reduced system 
stability, the data were beset with standing wave issues. Even though the gas cell test data were corrected from spectral baseline distortion, the extracted sideband ratio still showed large variations. Therefore, we believe that the sideband ratio measured during the gas cell tests in the HIFI bands 6 and 7 is not representative of the true mixer response in those bands and should be ignored for future error budgets. Further data mining will be undertaken during the post operation period to confirm that the sideband ratio across the HEB bands is 0.5. All evidence so far supports this assumption.

\section{Discussion and lessons learned}

The gas cell test campaign provided thousands of measurement points prior to the launch of Herschel, building probably one of the largest dataset dedicated to the calibration of the sideband ratio in a double sideband receiver, and demonstrating the unique capabilities of HIFI as a high-resolution spectrometer already years before the Herschel launch. Yet, more than half a year after the mission completion the sideband ratio correction is still puzzling in several areas of the HIFI frequency range. One of the main reasons for this is the relative scarcity of measurement points that was inherent to the limited number of gases available for the gas cell tests. This frequency coverage granularity later proved to be insufficient in bands such as band 1 or 2 where steep sideband ratio variations have been confirmed to occur over relatively narrow frequency domains.

The best way to join the dots between the pre-launch measurement points is to make use of high signal-to-noise spectral sweeps that can provide relative variation of line intensities within frequency steps of a few hundred $\mathrm{MHz}$. The combination of the absolute sideband ratio points derived from the gas cell tests, and their relative evolution in flight data is therefore needed to derive the detailed structure of the gain profile over the whole HIFI range. This approach was considered before launch already when a full spectral scan of the methanol molecule was collected in the laboratory. While this dataset probably contains most of the answers we are after, it is to date still too complex to interpret due to often non-mature spectroscopic parameters (e.g. pressure broadening) for methanol, which is a mandatory input for a correct modelling of the molecular absorption (most of it is non-saturated) as a function of the frequency.

We note that part of the complexity uncovered in e.g. band 1 was not anticipated, even during the design of the mixer circuits. From the early FTS measurements of the mixer broadband coupling, it was unclear that significant variations of the sideband ratio over frequency ranges as narrow as $4 \mathrm{GHz}$, such as the one evidenced e.g. around the ${ }^{12} \mathrm{CO} 5-4$ line (Fig. 6), would apply to the mixer response. In hindsight OCS measurements on a finer LO frequency grid would have helped unravel the coupling profile of bands 1 and 2. It is still unclear whether the discrepancy between those FTS measurements and the gas cell test data is simply due to the fact that the FTS tests did not use pumped mixers. We suggest that this discrepancy could be further investigated by groups involved in heterodyne detector design.

One of the important questions for the astronomers is, what is the final absolute accuracy of the line intensity observed, which for HIFI often boils down to the contribution of the sideband ratio. Because the sideband ratio is currently assumed 
to be constant over most of the tuning range, the same is true for its accuracy, which is simply taken as the standard deviation of the sideband gains derived from the gas cell tests over a given band. We discussed in Section 5.5 that this is too pessimistic e.g. for the HEB bands. On top of that, our description of the sideband ratio is LO- and IF-dependent, hence the calibration accuracy should be treated accordingly. Other aspects of the sideband ratio calibration, such as standing wave and/or diplexer cross-talk (not discussed in detail in this paper), are too complex to measure at such granularity. This is where the need of a detailed instrument model becomes important, and such work is currently on-going within the HIFI team [5]. It is unrealistic to assume that a theoretical model could accurately predict the frequency-dependent sideband ratio profile at any given LO frequency, so the idea here is not to correct observational data by synthetic instrument response function. Rather, such a model should provide the order of magnitude of typical calibration errors resulting from optical effects such as standing waves, esp. in the diplexer bands.

An important lesson from the sideband ratio calibration effort is that a large fraction of the answers can be directly extracted from the science data themselves. While the gas cell test campaign offered measurement conditions with a fully controlled sky signal input, it was relatively constrained in time and performed at a moment when the instrument understanding was not fully mature, so some of the data peculiarities where still uncovered or not accounted for at the time. The regular science observations gathered over the almost four years of mission provide the complementary information that not only allows to revisit some of the laboratory data, but also to probe the instrument behaviour in an often larger variety of parameter space than could be considered pre-launch. The combination of the two is the key to build the most accurate picture of the receiver characteristics, and offer the best possible calibration to the legacy archive data.

In that respect, the main source of information lies in the so-called spectral surveys, where a given spectral range is observed several times at nearby LO frequency tunings. This redundancy allows to invert the Double Sideband problem and build a Single Sideband solution - this is called deconvolution [1]. In this process, the gains applying respectively to the LSB and USB can be fitted in order to reconcile all individual DSB spectra with the SSB solution. Intrinsically the sideband gain profile inferred from this algorithm is only relative and needs to be tied to absolute values measured somewhere else (typically during the gas cell tests). The quality of the recovered sideband gain profile depends on the line density (if it is too large the risk of line blend is high, if it is too low the problem becomes degenerated at some frequencies). The HIFI team is currently running simulations on synthetic data with a variety of user-fed sideband ratio models and line density to identify the best data-set of spectral surveys to be used for that purpose, together with the accuracy associated with such an approach. The goal will be to extract an as continuous as possible profile of the sideband ratio over the HIFI observational range. Until then, the recommendation is to use the sideband ratio measured at particular line frequencies during the gas cell test campaign. This information, along with the calibration uncertainty associated with the sideband ratio, is communicated via the technical note[28] found on the HIFI calibration webpages.

\footnotetext{
${ }^{2}$ http://herschel.esac.esa.int/twiki/pub/Public/HifiCalibrationWeb/
} 
Acknowledgements We would like to thank Mihkel Kama from the CHESS team for his help in investigating the sideband ratio effect around the $557 \mathrm{GHz}$ water line. Ronan Higgins would like to thank Netty Honingh for useful discussions on the origins of the sideband ratio imbalance in bands 1 and 2. The authors are grateful to the anonymous referee for valuable comments.

\section{References}

1. Comito, C., \& Schilke, P. 2002, A\&A, 395, 357

2. Cherednichenko, S., Drakinskiy, V., Berg, T., Khosropanah, P., \& Kollberg, E. 2008, Review of Scientific Instruments, 79, 034501

3. de Graauw, T., Helmich, F. P., Phillips, T. G., et al. 2010, A\&A, 518, L6+

4. de Lange, G., Jackson, B. D., Eggens, M., et al. 2003, in Fourteenth International Symposium on Space Terahertz Technology, ed. C. Walker \& J. Payne, $447-+$

5. Delforge, B., Pearson, J., Roelfsema, P., Verheijen, M., \& Jellema, W. 2013, in 24th International Symposium of Space Terahertz Technology, Groningen, the Netherlands

6. Delorme, Y., Salez, M., Lecomte, B., et al. 2005, in Sixteenth International Symposium on Space Terahertz Technology, 444-448

7. Dieleman, P., Luinge, W., Whyborn, N. D., et al. 2008, Ninteenth International Symposium on Space Terahertz Technology, 106

8. Griffin, M. J., Abergel, A., Abreu, A., et al. 2010, A\&A, 518, L3+

9. Guan, X., Stutzki, J., Graf, U. U., et al. 2012, A\&A, 542, L4

10. Higgins, D. 2011, PhD thesis, National University of Ireland, Maynooth

11. Higgins, R. D. \& Kooi, J. W. 2009, in Society of Photo-Optical Instrumentation Engineers (SPIE) Conference Series, Vol. 7215, Society of Photo-Optical Instrumentation Engineers (SPIE) Conference Series

12. Higgins, R. D., Teyssier, D., Pearson, J. C., Risacher, C., \& Trappe, N. A. 2010, in Twenty-First International Symposium on Space Terahertz Technology, 390-397

13. Ian Avruch, Sylvie Beaulieu, Adwin Boogert, et al. 2013, The HIFI Data Reduction Guide, Tech. rep., ESAC

14. Kama, M., López-Sepulcre, A., Dominik, C., et al. 2013, A\&A, 556, A57

15. Karpov, A., Miller, D., Rice, F., et al. 2007, Applied Superconductivity, IEEE Transactions on, 17, 343

16. Kooi, J. \& Ossenkopf, V. 2009, in 20th International Symposium of Space Terahertz Technology, Charlottesville, Virginia, 69

17. Lis, D., Phillips, T., Goldsmith, P., et al. 2010, A\&A, 521

18. Mangum, J. 2002, ALMA Memo 434

19. Mueller, M., Jellema, W., Delforge, B., et al. 2013, "Experimental Astronomy", this Volume

20. Ossenkopf, V. 2003, ALMA Memo 442

21. Pilbratt, G. L., Riedinger, J. R., Passvogel, T., et al. 2010, A\&A, 518, L1+

22. Poglitsch, A., Waelkens, C., Geis, N., et al. 2010, A\&A, 518, L2+

23. Roelfsema, P. R., Helmich, F. P., Teyssier, D., et al. 2012, A\&A, 537, A17

24. Siebertz, O., Honingh, C., Tils, T., et al. 2007, in 18th International Symposium of Space Terahertz Technology, California Institute of Technology, Pasadena, California, 117-122 
25. Teipen, R., Justen, M., Tils, T., et al. 2005, in Sixteenth International Symposium on Space Terahertz Technology, 199-204

26. Teyssier, D., Dartois, E., Deboffle, D., et al. 2004, in 15th International Symposium of Space Terahertz Technology, Northampton, Massachusetts, 306-312

27. Teyssier, D., Whyborn, N. D., Luinge, W., et al. 2008, in Ninteenth International Symposium on Space Terahertz Technology, ed. W. Wild, 132-+

28. Teyssier, D., \& Higgins, R., 2013, Technical note: Sideband Ratio correction for HIFI data, HIFI calibration wiki page 